La identidad estudiantil y sentido de pertenencia que presentan los alumnos de la División Académica de Ciencias Económico Administrativas (DACEA) de la Universidad Juárez Autónoma de Tabasco (UJAT).

\title{
LA IDENTIDAD ESTUDIANTIL Y SENTIDO DE PERTENENCIA QUE PRESENTAN LOS ALUMNOS DE LA DIVISIÓN ACADÉMICA DE CIENCIAS ECONÓMICO ADMINISTRATIVAS (DACEA), DE LA UNIVERSIDAD JUÁREZ AUTÓNOMA DE TABASCO (UJAT)
}

\section{STUDENT IDENTITY AND SENSE OF BELONGING THAT STUDENTS SHOW IN DIVISION ACADEMICA DE CIENCIAS ECONOMICO ADMINISTRATIVAS (DACEA) FROM UNIVERSIDAD JUAREA AUTONOMA DE TABASCO (UJAT)}

\author{
Pamela Puig Hernández*, Leticia Rodríguez Ocaña**, \\ Herminia Banda Izeta***
}

\begin{abstract}
*Pasante de la Licenciatura de Mercadotecnia. División Académica de Ciencias Económico Administrativas de la Universidad Juárez Autónoma de Tabasco. ORCID: https://orcid.org/0000-0002-2401-172X.

**Doctora en Antropología en Situaciones de Riego. Profesor Investigador de la División Académica de Ciencias Económico Administrativas, Universidad Juárez Autónoma de Tabasco. Email: letiro6@hotmail.com. ORCID: https://orcid.org/0000-0003-0491-2886.

***Doctora en Educación Superior y Mercadotecnia en Negocios Turísticos. Profesor Investigador de la División Académica de Ciencias Económico Administrativas, Universidad Juárez Autónoma de Tabasco. Email: hbi05@hotmail.com. ORCID: https://orcid.org/0000-0003-4565-3577.
\end{abstract}

Dirección para recibir correspondencia: pamela_puig@hotmail.com

Fecha de recibido: 12 de mayo de 2019

Fecha de aceptación: 20 de junio de 2019

DOI: https://doi.org/10.19136/hitos.a25n72.3465

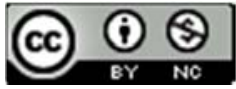

PUIG-HERNÁNDEZ P., RODRÍGUEZ-OCAÑA L., BANDA-IZETA H.

MAYO-AGOSTO 2019. Año 25, Número 72. Págs. 300-312 
La identidad estudiantil y sentido de pertenencia que presentan los alumnos de la División Académica de Ciencias Económico Administrativas (DACEA) de la Universidad Juárez Autónoma de Tabasco (UJAT).

\section{RESUMEN}

OBJETIVO: Conocer la identidad estudiantil y sentido de pertenencia que presentan los alumnos de la División Académica de Ciencias Económicas Administrativas (DACEA), de la Universidad Juárez Autónoma de Tabasco (UJAT).

MATERIAL Y MÉTODO: Investigación cuantitativa, descriptivo. La población se determinó tomando en cuenta las matriculas de las cuatro Licenciaturas de la DACEA. Con un total de 4,552 Estudiantes (2018). En base a la muestra poblacional obtenida, se aplicaron 354 cuestionarios, teniendo un nivel de confianza del 95\%, el instrumento de recolección cuenta con un total de 16 reactivos positivos, mismos que fueron tomados de la investigación Escala de Identidad Universitaria, de Almazán, Camacho y García (2014).

RESULTADOS: En la investigación sobre la identidad universitaria realizada a los alumnos de las cuatro licenciaturas, se obtuvo como resultado que las licenciaturas con mayor grado de identidad universitaria y sentido de pertenencia fueron las de Mercadotecnia y Administración.

CONCLUSIONES: Los estudiantes se sienten parte de la universidad, deseaban estar en la institución, es mejor la institución de ahora que donde se encontraban antes, cuidan las instalaciones, se esforzaron para poder ingresar a la universidad, conocieron a sus mejores amigos en la institución, pero no conocen el reglamento de la institución.

PALABRAS CLAVE: Identidad universitaria. Percepción de la universidad. Población estudiantil. Sentido de pertenencia.

\section{ABSTRACT}

OBJECTIVE: To know student identity and sense OF belonging that students show in Division Academica de Ciencias Economico Administrativas (DACEA) at Universidad Juarez Autonoma de Tabasco (UJAT).

MATERIAL AND METHOD: Quantitative research of descriptive type. The population was determined considering the enrollment in all four careers in DACEA, with a total of 4,552 students. Based on the obtained sample, 354 questionnaires were administered, with a 95\% reliabilty, the 
La identidad estudiantil y sentido de pertenencia que presentan los alumnos de la División Académica de Ciencias Económico Administrativas (DACEA) de la Universidad Juárez Autónoma de Tabasco (UJAT).

instrument consists of 16 positive items from the University Identity Scale from Almazán, Camacho \& Garcia's study (2014).

RESULTS: From the research conducted in all four careers, results indicate that Administration and Marketing students show a higher degree of university identity and sense of belonging.

CONCLUSIONS: Students feel part of the university, they longed to be in the institution, it is a better choice from where they used to be, facilities are maintained, effort was made to become a student, best friends were met but university policies are unknown to them.

KEY WORDS: University identity. University perception. Student population. Sense of belonging.

\section{INTRODUCCIÓN}

La universidad es una parte crucial para la vida de todo individuo para su formación personal y académica. Estar en una institución académica como la universidad, no sólo es asistir a clases, conforme al paso de los semestres o años escolares, te hace sentir parte de la misma, ya sea por la convivencia o el tiempo que se comparte entre compañeros, profesores y trabajadores administrativos; de tal manera que, existe un mejor aprovechamiento de cada integrante que conforma la universidad.

\section{MARCO TEÓRICO}

\section{Concepto Identidad}

"La identidad no es otra cosa que el resultado estable y provisorio, individual y colectivo, subjetivo y objetivo, biográfico y estructural de los diversos procesos de socialización que, conjuntamente, construyen los individuos y definen las instituciones" Dubar (2001), citado por Molina (2015).

\section{Identidad Universitaria}

Existen factores tangibles e intangibles que propician la identidad universitaria, como son: el escudo, el himno, botargas representativas (mascota), uniformes, talleres culturales y actividades deportivas, áreas comunes, visión, misión, objetivos institucionales, valores, entre otros (Rodríguez, 2013). 
La identidad estudiantil y sentido de pertenencia que presentan los alumnos de la División Académica de Ciencias Económico Administrativas (DACEA) de la Universidad Juárez Autónoma de Tabasco (UJAT).

"En su mayor parte, los jóvenes, hombres y mujeres, de este final de siglo crecen en una suerte de presente permanente sin relación orgánica alguna con el pasado del tiempo en el que viven" (Hobsbawm, 1995).

Actualmente, tener una buena identidad universitaria es muy complejo ya que en cada década ha sido muy diferente, en la actualidad existen diversas plataformas digitales que permiten estar en sistemas educativos virtualmente, eso ha hecho que los estudiantes sean muy individualistas y por lo mismo no existe un vínculo e identidad tan estrecha como lo era antes.

\section{Concepto de Sentido de Pertenencia}

“a) El hombre es un animal con deseos; es decir, que alguna necesidad domina siempre su conducta; b) Existe una jerarquía de preponderancia en las necesidades del hombre, y c) Una necesidad satisfecha ya no causa motivación" Maslow (1943).

Además de la identidad, la percepción es otro elemento muy importante en este proceso de la formación del individuo o persona y la institución, la percepción es la manera en la cual las personas ven y perciben las cosas de diferente manera conforme a sus principios y experiencias mediante sus cinco sentidos que van de la mano con las vivencias que han tenido a lo largo de su vida, así como los diferentes factores que conforman la identidad universitaria según Escala de Identidad Estudiantil Universitaria de Cabral (2006) (ver tabla 1).

\section{Tabla 1}

\section{Escala de identidad estudiantil}

\begin{tabular}{ll}
\hline Relaciones humanas: Comprenden la & Afinidades: Son intereses mutuos y sirven para \\
interacción entre uno o más individuos donde & formar parte de un grupo con los mismos \\
interactúan y comparten información. & gustos.
\end{tabular}

Pertenencia: Que forma un vínculo con Educación: Un proceso de socialización de los individuos y tener una convivencia da sentido individuos, también implica una conciencia claro de pertenencia como afectivo y psicológico. cultural, conductual; una serie de habilidades y valores que formaran al individuo en una persona integral.

Percepción de la universidad como unidad: Es la Vínculo: La relación de un individuo con la forma como todos los integrantes de una universidad perciben su institución como un todo. institución -compañeros, profesores, trabajadores y funcionarios administrativos- $y$ continuar con ese vínculo toda la vida 
La identidad estudiantil y sentido de pertenencia que presentan los alumnos de la División Académica de Ciencias Económico Administrativas (DACEA) de la Universidad Juárez Autónoma de Tabasco (UJAT).

Territorialidad: Es la forma de cada grupo social, Reglas: Son las normas que se deben de que pueda reconocer su territorialidad e respetar por medio de un acuerdo y que indican identificarse con la misma respetando su el comportamiento de cada individuo en un entorno, reglamento y valores para dar seguridad grupo, o bien en dicha institución.

y sentido de pertenecía.

Experiencia escolar pasada: Son experiencias o vivencias de cada individuo en su vida escolar o académica y que son las bases fundamentales para comprender o entender al sujeto sus vínculos con él y con la institución.

Fuente: Escala de Identidad Estudiantil de Cabral y Col (2006).

Dificultad de ingreso la institución: Son las circunstancias y como adaptarse a ellas en la nueva institución.

"Es el proceso por medio del cual uno adquiere el significado de la identidad personal y aprende lo que las personas en la cultura circundante creen y como esperan que uno se comporte" (Mastretta, 2008).

\section{MATERIALES Y MÉTODOS}

La metodología para el desarrollo de esta investigación parte de un enfoque cuantitativo descriptivo. Para la extraccion de la muestra, se utilizo la técnica de muestreo probabilístico aleatorio simple, obteniendose un total de 354 estudiantes, en la División Académica de Ciencias Económico Administrativas (DACEA), utilizando como instrumento de recolección de datos las siguientes técnicas: revisión documental, observación y encuesta (de acuerdo a la escala de Likert).

El diseño de estudio utilizado para esta investigación fue no experimental, porque se realizó sin la manipulación deliberada de variables, en los que soló se observaron los fenomenos en su ambiente natural, para después analizarlos y transversal porque la recolección de los datos se da en solo un momento (Hernández-Sampieri, Fernández y Baplista, 2010). 
La identidad estudiantil y sentido de pertenencia que presentan los alumnos de la División Académica de Ciencias Económico Administrativas (DACEA) de la Universidad Juárez Autónoma de Tabasco (UJAT).

\section{Población}

La DACEA de la Universidad Juárez Autónoma de Tabasco (UJAT), esta cituada en la ciudad de Villahermosa, Tabasco, integrada por una población de 4,552 alumnos, inscritos en cuatro licenciaturas, en la modalidad presencial.

En base a la muestra poblacional obtenida, se aplicaron 354 cuestionarios, teniendo un nivel de confianza del 95\%, el instrumento de recolección cuenta con un total de 16 reactivos positivos, mismos que fueron tomados de la investigación Escala de Identidad Universitaria (EIEU), de Almazán, Camacho y García (2014), usando los parámetros:

- Totalmente de acuerdo.

- De acuerdo.

- En desacuerdo.

- Totalmente de acuerdo.

Para conocer el grado de identidad, en base a la EIEU (Cabral, Escala de Identidad Estudiantil Universitaria., 2006) a los alumnos de las licenciaturas de Mercadotecnia, Economía, Contaduría Pública y Administración de la DACEA-UJAT del $4^{\circ}$ al $10^{\circ}$ semestre, Se obtuvieron los datos proporcionados en la "Matrícula Escolar por Programa Educativo y Tipo de Ingreso de Licenciatura" en el 3er Informe de Actividades 2019.

\section{RESULTADOS}

Los resultados del estudio muestran la participación de 354 alumnos de $4^{\circ}$ a $10^{\circ}$ semestre de las cuatro licenciaturas, de acuerdo a los porcentajes obtenidos, las licenciaturas que tienen un mayor índice de población estudiantil fueron las de Administración y Mercadotecnia (ver figura 1), el número de mujeres fue mayor que el de los hombres, la edad promedio de la población estudiantil fue de 24 años, la mayoría de las mujeres encuestadas son solteras y no trabajan, se dedican en su mayoría del tiempo a la universidad. 
La identidad estudiantil y sentido de pertenencia que presentan los alumnos de la División Académica de Ciencias Económico Administrativas (DACEA) de la Universidad Juárez Autónoma de Tabasco (UJAT).

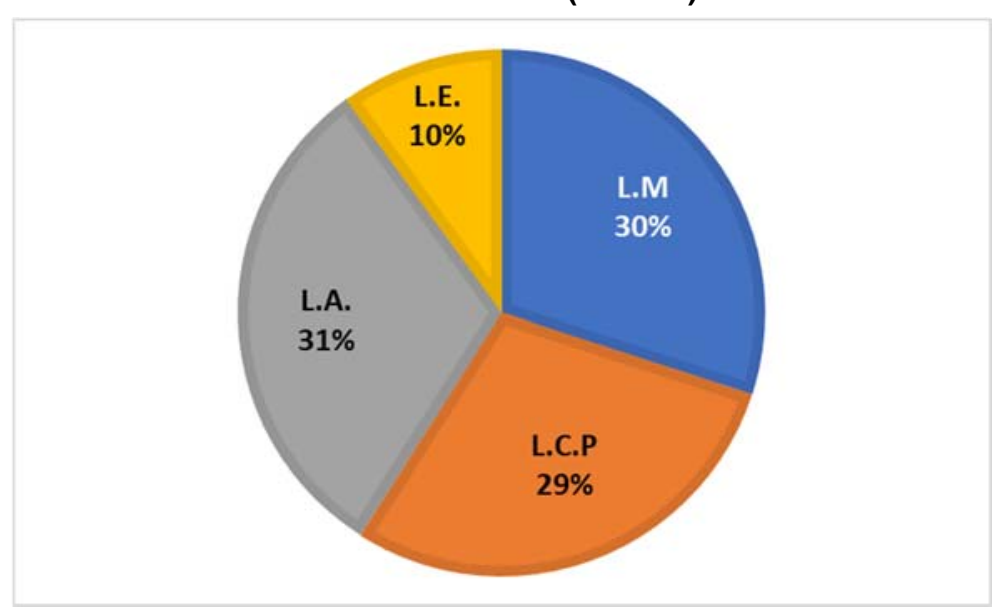

\section{Figura 1. Licenciaturas encuestadas.}

Fuente: Elaboración propia.

El resultado porcentual de las cuatro licenciaturas de la DACEA-UJAT que tienen más población estudiantil, son: 31\% alumnos de la Licenciatura de Administración, seguidos por un 30\% de los alumnos de Mercadotecnia, el 29\% alumnos de Contaduría Pública y $10 \%$ de los estudiantes de la Licenciatura de Economía.

Por lo que respecta a los semestres con mayor índice de identidad entre la población estudiantil, se muestra que el $24 \%$ son del $10^{\circ}$ semestre, seguido por un $21 \%$ son del $9^{\circ}$ y $21 \% 8^{\circ}$ semestre, un $15 \%$ son de $7^{\circ}$ semestre, el $13 \%$ son del $6^{\circ}$ semestre, el $3 \%$ restante son de los estudiantes del $4^{\circ}$ y $3 \% 5^{\circ}$ semestre.

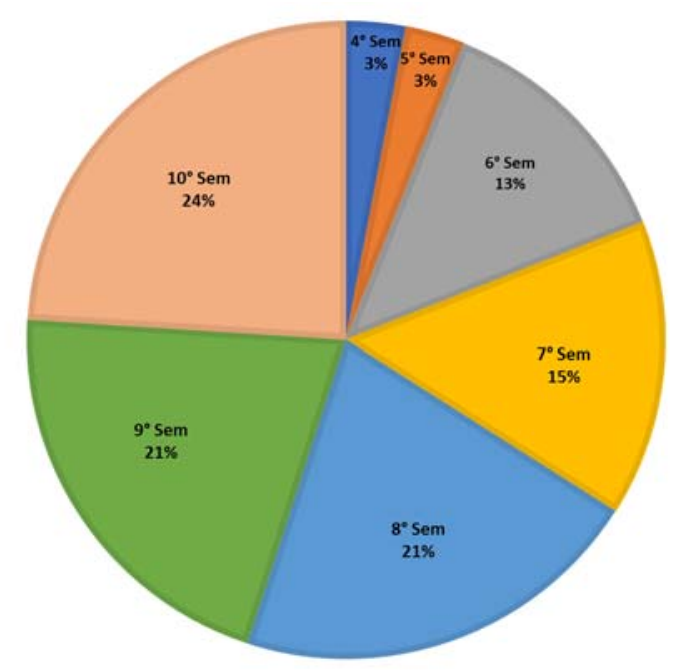

Figura 2. Semestre con mayor índice de identidad.

Fuente: Elaboración propia. 
La identidad estudiantil y sentido de pertenencia que presentan los alumnos de la División Académica de Ciencias Económico Administrativas (DACEA) de la Universidad Juárez Autónoma de Tabasco (UJAT).

Entre la población encuestada, se encontró que el 45\% tienen 24 años, un 35\% son de 23 años, seguidos por un $14 \%$ de 22 años, el 1\% restante en el rango de 25 años, $1 \%$ de 26 años, 1\% de 27 años, $1 \%$ de 28 años, 1\% de 30 años, 1\% de 36 años.

De los 354 alumnos encuestados, se encontró que 52\% fueron mujeres y el $48 \%$ hombres (ver figura 3), el $68 \%$ de los estudiantes no trabaja mientras el $32 \%$ si cuentan con un trabajo. Del total de los encuestados, el $76 \%$ son solteros seguido por un $18 \%$ en unión libre y el $6 \%$ separados.

Figura 3. Sexo de los estudiantes.

Fuente: Elaboración propia.

Además de los aspectos generales, existen otros factores importantes para la identidad universitaria según Escala de identidad Estudiantil Universitaria de Cabral, et.al (2006) que son:

1) Relaciones humanas.

2) Afinidades.

3) Pertenencia.

4) Educación.

5) Percepción de la universidad como unidad.

6) Vínculo.

7) Territorialidad.

8) Reglas.

9) Experiencia escolar pasada.

10) Dificultad de ingreso a la institución. 
La identidad estudiantil y sentido de pertenencia que presentan los alumnos de la División Académica de Ciencias Económico Administrativas (DACEA) de la Universidad Juárez Autónoma de Tabasco (UJAT).

Los resultados expuestos demuestran que el $57 \%$ de los alumnos se sienten parte de la universidad desde su ingreso, (ver tabla 2).

\section{Tabla 2}

¿Me siento parte de la universidad desde que ingresé?

\begin{tabular}{ll}
\hline Respuesta & Porcentajes \\
\hline Totalmente de acuerdo & $21 \%$ \\
De acuerdo & $36 \%$ \\
En desacuerdo & $23 \%$ \\
Totalmente desacuerdo & $20 \%$ \\
\hline
\end{tabular}

Fuente: Elaboración propia.

Con respecto a la pregunta, ¿Me siento feliz cuando se menciona el nombre de la universidad a la que pertenezco?, el 37\% del total de los encuestados está en desacuerdo, seguidos por 32\% de acuerdo, el $20 \%$ restante totalmente de acuerdo y un $11 \%$ totalmente de desacuerdo.

En la pregunta, ¿Cuándo estuve en otras instituciones deseaba a esta institución? el 40\% está de acuerdo, seguidos por $30 \%$ en desacuerdo, el $18 \%$ restante totalmente de desacuerdo y un $12 \%$ totalmente de acuerdo.

Los resultados de la pregunta ¿Creo que la institución donde me encuentro hoy es mejor que donde me encontraba?, se obtuvo un 33\% de acuerdo, seguidos por $28 \%$ en totalmente de acuerdo, el $22 \%$ en desacuerdo y un $17 \%$ totalmente desacuerdo. Los alumnos defienden la institución ante comentarios negativos de los demás, el 41\% la defiende, (ver tabla 3).

\section{Tabla 3}

¿Defiendo mi institución ante comentarios negativos de los demás?

\begin{tabular}{ll}
\hline Respuesta & Porcentajes \\
\hline Totalmente de acuerdo & $15 \%$ \\
De acuerdo & $26 \%$ \\
En desacuerdo & $26 \%$ \\
Totalmente desacuerdo & $33 \%$ \\
\hline
\end{tabular}

Fuente: Elaboración propia. 
La identidad estudiantil y sentido de pertenencia que presentan los alumnos de la División Académica de Ciencias Económico Administrativas (DACEA) de la Universidad Juárez Autónoma de Tabasco (UJAT).

Los estudiantes se sientes orgullosos de pertenecer a la institución donde se encuentran, (ver tabla 4), tienen un total aprecio especial por la institución donde pertenecen (ver tabla 5) y el 40\% se sienten vinculados positivamente a la institución donde pertenecen y el $88 \%$ de los estudiantes se sienten orgullosos de que haya entrado con mis propios méritos.

\section{Tabla 4}

¿Me siento orgulloso de pertenecer a la institución donde me encuentro?

\begin{tabular}{ll}
\hline Respuesta & Porcentajes \\
\hline Totalmente de acuerdo & $20 \%$ \\
De acuerdo & $40 \%$ \\
En desacuerdo & $27 \%$ \\
Totalmente desacuerdo & $13 \%$
\end{tabular}

Fuente: Elaboración propia.

\section{Tabla 5}

¿Tengo un aprecio especial por la institución donde pertenezco?

\begin{tabular}{ll}
\hline Respuesta & Porcentajes \\
\hline (4) Totalmente de acuerdo & $37 \%$ \\
(3) De acuerdo & $23 \%$ \\
(2) En desacuerdo & $18 \%$ \\
(1) Totalmente desacuerdo & $22 \%$ \\
\hline
\end{tabular}

Fuente: Elaboración propia.

En la tabla 6 , se encontró que el $75 \%$ de los alumnos no conoce el reglamento de la institución a la que pertenece.

\section{Tabla 6}

¿Conozco el reglamento de la institución a la que pertenezco?

\begin{tabular}{ll}
\hline Respuesta & Porcentajes \\
\hline Totalmente de acuerdo & $12 \%$ \\
De acuerdo & $13 \%$ \\
En desacuerdo & $30 \%$ \\
Totalmente desacuerdo & $45 \%$ \\
\hline
\end{tabular}

Fuente: Elaboración propia.

PUIG-HERNÁNDEZ P., RODRÍGUEZ-OCAÑA L., BANDA-IZETA H. 
La identidad estudiantil y sentido de pertenencia que presentan los alumnos de la División Académica de Ciencias Económico Administrativas (DACEA) de la Universidad Juárez Autónoma de Tabasco (UJAT).

\section{CONCLUSIONES}

Los estudiantes matriculados en la DACEA, brindan una serie de respuestas posibles en función de su proyecto personal en el contexto universitario. Los aspectos de este contexto sociocultural, determinan las formas particulares y colectivas del proceso de una construcción de identidad.

En base a los cuestionarios realizados: los estudiantes se sienten parte de la universidad, deseaban estar en la institución, es mejor la institución de ahora que donde se encontraban antes, cuidan las instalaciones, se esforzaron para poder ingresar a la universidad, conocieron a sus mejores amigos en la institución, pero no conocen el reglamento de la institución.

Los datos obtenidos demuestran en los aspectos intangibles, que los estudiantes se sienten felices y orgullosos cuando se menciona el nombre de la institución, y de pertenecer a ella, tienen un aprecio especial y se sienten vinculados a la institución, y orgullosos por entrar por sus propios méritos, no defienden a la institución en comentarios negativos y su comportamiento no sería diferente si pertenecieran a otra institución.

Si bien, en las definiciones que se mencionaron al principio de la investigación el resultado de un grado medio de sentido de pertenencia e identidad universitaria. Cabe mencionar, que este porcentaje puede y debe elevarse, mediante acciones que coadyuven en un alto grado de Identidad Universitaria, como podría ser eventos obligatorios culturales, sociales y deportivos.

El determinar el grado de identidad de en una institución académica en este caso la UJAT, es muy importante, ya que permite conocer, percibir y saber qué tanto los alumnos, profesores, administrativos y personas que conforman e integran la universidad se sientan identificados dentro y fuera de la institución y saber qué factores destacan de una universidad a otra, además de que la identidad universitaria va de la mano con la imagen de cualquier institución.

Los alumnos de la DACEA-UJAT, son Juchimanes por convicción, muy orgullosos y satisfechos por cada experiencia y vivencia que han vivido en la universidad, la cual está formando como profesionales para el futuro de cada uno de ellos, agradeciendo a sus profesores que instruyeron a lo largo de los años de su carrera siempre recordando su vida universitaria, como una de las mejores etapas de sus vidas y queda por hacer profesionistas, y llevar en alto el nombre de la universidad en todos los ámbitos que se les presente en su vida profesional y personal. 
La identidad estudiantil y sentido de pertenencia que presentan los alumnos de la División Académica de Ciencias Económico Administrativas (DACEA) de la Universidad Juárez Autónoma de Tabasco (UJAT).

\section{REFERENCIAS BIBLIOGRÁFICAS}

Barthey, S. H. (1982). Principios de percepción. México D. F.: Trillas.

Brubaker, R. (2001). Más allá de la identidad. Apuntes de investigación del CECYP, 7, 21-65.

Cabral, M. V. (2006). Escala de identidad estudiantil universitaria. Identidad estudiantil universitaria en estudiantes de licenciatura.

Cabral, M. V. (2006). Identidad estudiantil universitaria en estudiantes de licenciatura. Revista Psicología Científica.com, 15.

Cappello, H. M. (2015). La identidad universitaria. La construcción del concepto. Revista Internacional de Ciencias Sociales y Humanidades, SOCIOTAM, XXV (2), 22-57.

Conrad, K. (2011). Antropología Cultural . México: McGraw-Hill.

Díaz, M., Quintero, A. \& Gutierrez, O. (08 de Febrero de 2010). Identidad. Recuperado de Ciaf.edu: http://www.ciaf.edu.co/ciem/proyectos/identidad_universitaria.pdf

Dubar, C. (2001). El trabajo y las identidades profesionales y personales. Latinoamericana de Estudios del Trabajo, 5 - 17.

Hernández, Fernández y Baptista (2010). Metodología de la investigación, ( $6^{a}$ ed.). México: McGraw-Hill.

Hobsbawm, E. (1995). Historia del siglo XX . Barcelona, España: Grijalbo Mondadori S.A.

I., M. (2004). La Identidad Universitaria. Mexico : La UAM- I y la UT- . Recuperado de La UAM- I y la UT.

Jenkins, R. (1996). Social identity theorising social identity. Inglaterra: Routledge.

LIC, A. L. (2006). La identidad Universitaria. La identidad Universitaria (p. 18). Estado de Mexico: S/E.

Maslow, A. (1943). A theory of human motivation. USA.

Mastretta, G. V. (2008). Sociología de la organización. México: Limusa.

Molina Benítez, J. A. (2015). Recorrido por dos ámbitos identitarios: universidad y ciberespacio. LASALLISTA de Investigación, 12(2), 204-214. Recuperado de http://www.redalyc.org/pdf/695/69542291021.pdf

Oviedo, G. (12 de 03 de 2019). La definición del concepto de percepción en psicología con base en la teoría gestalt. Recuperado de Blog da Psicologia da Educação: https://www.ufrgs.br/psicoeduc/gestalt/percepcion-en-la-teoria-gestalt

Puga Cristina, P. J. (2007). Hacia la Sociología. México: Pearson.

Robledo, J. R. (S/A). La Identidad Universitaria y la Integración Social. Identidad BUAP e integración social, (p. 12). Puebla, México.

Santiago, Z. (2012). Aspectos socioeconómicos de la problemática en México. México: Limusa.

PUIG-HERNÁNDEZ P., RODRÍGUEZ-OCAÑA L., BANDA-IZETA H.

MAYO-AGOSTO 2019. Año 25, Número 72. Págs. 300-312 
La identidad estudiantil y sentido de pertenencia que presentan los alumnos de la División Académica de Ciencias Económico Administrativas (DACEA) de la Universidad Juárez Autónoma de Tabasco (UJAT).

Tere Almazán Mason, J. C. (2014). Escala de identidad universitaria. México: Facultad de Psicología, UNAM.

Universidad Juárez Autónoma de Tabasco. (13 de 03 de 2019). Recuperado de http://www.archivos.ujat.mx/2019/rectoria/3ER-INFORME-DE-ACTIVIDADES-2018.pdf

Vargas Perez, M. (12 de 01 de 19). Recuperado de Estudio Sobre la identidad universitaria en egresadosde la UPN Ajusco: http://200.23.113.51/pdf/27929.pdf

Zepeda, A. V. (22 de Marzo de 2013). La gaceta. Recuperado de http://gaceta.udg.mx/G_nota1.php?id=13427 